\title{
WILEY-VCH
}

DOI: $10.1002 /(($ please add manuscript number $))$

Article type: Communication

\section{Universal Soft Robotic Microgripper}

Haiyan Jia, Erik Mailand, Jiangtao Zhou, Zhangjun Huang, Giovanni Dietler, John M. Kolinski, Xinling Wang* and Mahmut Selman Sakar*

Haiyan Jia, Erik Mailand, Prof. John M. Kolinski, Prof. Mahmut Selman Sakar Institute of Mechanical Engineering

Ecole Polytechnique Fédérale de Lausanne

CH-1015 Lausanne, Switzerland.

E-mail: selman.sakar@epfl.ch

Haiyan Jia, Prof. Xinling Wang

School of Chemistry and Chemical Engineering

State Key Laboratory of Metal Matrix Composites

Shanghai Jiao Tong University

Dongchuan Road No. 800, Shanghai 200240, China

E-mail: xlwang@sjitu.edu.cn

Jiangtao Zhou, Prof. Giovanni Dietler

Laboratory of Physics of Living Matter

Ecole Polytechnique Fédérale de Lausanne

$\mathrm{CH}-1015$ Lausanne, Switzerland

Dr. Zhangjun Huang

Institut des Sciences et Ingénierie Chimiques

Ecole Polytechnique Fédérale de Lausanne

CH-1015 Lausanne, Switzerland

Keywords: soft robotics, biomanipulation, smart materials, microfabrication 


\section{WILEY-VCH}

We present a soft robotic microgripper that consists of a smart actuated microgel connected to a spatially photopatterned multifunctional base. When pressed onto a target object, the microgel component conforms to its shape, thus providing a simple and adaptive solution for versatile micromanipulation. Without the need for active visual or force feedback, objects of widely varying mechanical and surface properties are reliably gripped through a combination of geometrical interlocking mechanisms instantiated by reversible shape-memory and thermal responsive swelling of the microgel. The gripper applies holding forces exceeding $400 \mu \mathrm{N}$, which is high enough to lift loads 1000 times heavier than the microgel. An untethered version of gripper is developed by remotely controlling the position using magnetic actuation and the contractile state of the microgel using plasmonic absorption. Gentle yet stable robotic manipulation of biological samples under physiological conditions opens up possibilities for high-throughput interrogation and minimally invasive interventions. 


\section{WILEY-VCH}

Machines that can be programmed to operate on biomedical samples with high dexterity and precision have the potential to become essential tools in many basic biological and clinical applications. ${ }^{[1,2]}$ Microengineered three-dimensional (3D) organotypic cultures have been gradually replacing more traditional assays on plastic dishes. ${ }^{[3,4]}$ The assembly, characterization, and testing of these constructs present fresh challenges for robotic biomanipulation. Physical phenomena playing negligible role in the operation of macroscale devices can have profound impact on the performance of microscale devices. For example, volume effects associated with inertia and body forces are dominated by surface effects associated with friction and adhesion forces. $^{[5]}$ As a result, microscale parts sculptured from bulk materials display limited functionality, particularly at the interface with organic components. Furthermore, living matter displays out-of-equilibrium dynamics by spontaneously changing the underlying morphology and mechanical properties in time. Soft robotic devices may effectively address these challenges through passive mechanical control mechanisms and distributed actuation. ${ }^{[6]}$ They provide safe interactions for both the machine and the sample because of a reduced stress concentration achieved through conformal contacts and high degrees of freedom. ${ }^{[7]}$ Hydrogels have emerged as a versatile substrate for engineering advanced biomedical microdevices because they allow rational design at the molecular level and control over multiscale architecture. ${ }^{[8]}$ They also possess dynamic mechanical functionalities under physiological conditions such as programmable shape change and self-healing. ${ }^{[9-13]}$

Robotic manipulation of small and delicate objects has been demonstrated either using actuated compliant end-effectors ${ }^{[14-17]}$ or rotors generating fluid flow. ${ }^{[18-20]}$ The prevailing strategy in contact micromanipulation is to design microgrippers that consist of actuated fingers that can reliably grasp objects. This paradigm has also been applied to engineer magnetically or optically controlled hydrogel microgrippers. ${ }^{[21-24]}$ Origami-inspired folding techniques are widely used for realizing hydrogel microgrippers due to the convenience of planar microfabrication 


\section{WILEY-VCH}

techniques, particularly photolithography. ${ }^{[12,25]}$ However, a multi-fingered microgripper is a complex system to build and control considering the vast diversity of shapes and mechanical properties of targeted biomedical payload, the lack of tactile sensors at small scale, and severely limited visual feedback at site of operation. Previous work introduced an alternative approach for handling macroscale objects, universal gripping, performed by an elastic bag filled with granular material that can autonomously conform to arbitrary shapes. ${ }^{[26]}$ This passive process has significant advantages in terms of reliability, cost, and speed. Although the concept is quite appealing, the presented realization is not practical for micromanipulation because it involves reversible jamming of particles by applying a vacuum. Building an analogous robotic device from hydrogels designed for microscale biomanipulation is the focus of this work.

In this communication, we introduce a universal soft robotic microgripper by utilizing programmable chemomechanical properties of double network hydrogels. The device was manufactured from a combination of natural and synthetic hydrogels. The gripping concept is illustrated in Figure 1a. The infinitely many degrees of freedom of this system are passively actuated upon contact with the surface of the objects. By molding itself around the cargo, the microgripper not only builds geometric constraints but also generates static friction from normal stresses at contact. This new configuration is locked in place by utilizing the shape-memory property of the microgel. Upon exposure to $\mathrm{Ca}^{2+}$, guluronic acid blocks on the sodium alginate ionically crosslink with each other via diffusing ions. The original state is recovered by either adding a chemical such as ethylenediaminetetraacetic acid (EDTA) that coordinates with $\mathrm{Ca}^{2+}$ or introducing ions such as $\mathrm{Na}^{+}$and $\mathrm{K}^{+}$that replace $\mathrm{Ca}^{2+}$ in the polymer chain. A second hydrogel, poly(N-isopropylacrylamide) (pNIPAM), serves as the structural backbone and introduces reversible swelling under the application of external stimulus. Contraction of pNIPAM tightens the contact between the gripper and the gripped object, and at the same time 


\section{WILEY-VCH}

hardens the microgel. Depending on the shape of the target object, actuation of the microgripper can also facilitate cargo delivery.

Smart microgels were fabricated using a microfluidic approach based on the breakoff process of droplets in two-phase flows (Figure 1b). A precursor solution of sodium alginate and NIPAM along with crosslinker and photoinitiator forms the discontinuous phase while the continuous phase comprises the surfactant and oil. The emulsion was exposed to UV light to initiate the free radical polymerization process. The microfluidic approach provides control over the size of microgels in the range of tens to hundreds of micrometers. The gripping performance depends on the synergistic contributions of sodium alginate and pNIPAM. We optimized the composition of the microgels in order to achieve a proper balance among mechanical strength (Figure S2), shape memory property (Figure S3), and reversible volumetric compaction (Figure S4). The contents were verified using Fourier transform infrared spectrum (Figure S5). Rotational rheometer measurements showed that the LCST of the microgels were $32^{\circ} \mathrm{C}$ as expected (Figure S6). Microgels were transferred to another microchannel filled with a polymer precursor solution, poly (ethylene glycol) diacrylate (PEGDA) mixed with photoinitiator, for the in situ polymerization of the gripper base. We chose PEGDA for manufacturing the gripper base due to its tunable stiffness, ease of polymerization, and insensitivity to temperature. A programmable digital mirror display (DMD) device illuminated the sample according to the blueprint without the need for a printed mask. We designed two versions of the base for the microgripper (denoted by Type 1 and 2) to be able to transport objects by either encapsulating the whole body or engaging with holes presented on their surface.

We evaluated the holding force of the microgripper by withdrawing a spherical object and monitoring the force required for withdrawal using a commercially available force sensor (Figure 2a). The spherical geometry of the sensor tip simplifies the analysis of the gripping 


\section{WILEY-VCH}

mechanism and allows direct comparison with model predictions. The Type 1 gripper used for these measurements was fixed to a flat surface. The force sensor was mounted on a 3-axis micromanipulator, and the workspace was monitored using an optical microscope (Figure S7). The spherical sensor tip was pressed on the microgel with a speed of $15 \mu \mathrm{m} \mathrm{s}^{-1}$ and then, once the compressive strain reached 0.6 , enough time ( $\sim 1$ minutes) was given for the hydrogel to relax. In a similar manner, the sensor tip was withdrawn from the gripper with the same speed until the contact was completely lost. The holding strength was denoted as the tensile force required to fully withdraw the sensor tip from the microgripper, and was recorded as the maximum positive value on the force curves shown in Figure 2. In the absence of $\mathrm{Ca}^{2+}$ and at room temperature, the holding force was almost zero as expected (Figure 2b). With the addition of $5 \mathrm{wt} \% \mathrm{Ca}^{2+}$, geometric interlocking generated a substantial holding force that was recorded as $100 \mu \mathrm{N}$ (Figure 2c). This force corresponds to the weight of a millimeter scale metal objects. Thus, the gripper can carry every microscale object it can grasp. As the temperature was increased above LCST, the volumetric contraction of the microgel resulted in a tighter grip on the sensor tip, leading to a significant increase in the holding strength that exceeds $400 \mu \mathrm{N}$ (Figure 2d). These values are comparable to the forces reported previously with MEMS-based microgrippers. ${ }^{[15,16]}$

We next studied how the magnitude of the holding force is influenced by the contact angle, degree to which the spherical object is enveloped by the gripper. Based on previous work on universal grippers, ${ }^{[26]}$ we hypothesized that the maximum holding force is primarily determined by the bending resistance of the material for contact angles higher than $90^{\circ}$. The force required to bend the ring wrapped around the sphere to vertical so the sphere can slip out is given by $F_{h}$ $\sim\left(2 \pi R t^{2} l l\right) \sigma$ where $l$ is the bending arm length, $t$ is the thickness of the gripper section, and $R$ is the radius of the sphere. Because $\mathrm{t}$ and 1 are comparable, taking $l \sim t \approx 50 \mu \mathrm{m}, \mathrm{R}=125 \mu \mathrm{m}$ 


\section{WILEY-VCH}

and $\sigma=2.3 \mathrm{kPa}$ from the AFM tests leads to $F_{h} \approx 100 \mu \mathrm{N}$. We see very good agreement between calculated and measured force, which verifies the dominant role of bending resistance. We developed finite element (FE) models of the microgripper to provide a means to predict the bending stress for different contact angles. Figure 2e illustrates the geometric parameters and boundary conditions. A prescribed displacement was directly applied to the arm in the normal direction and the equilibrium stress was simulated. The microgels were assigned the measured values of the modulus of $2.3 \mathrm{kPa}$ and the holding force was calculated by integrating the stress over the bending area. Figure $2 \mathrm{f}$ shows the results of the two-dimensional FE simulations. The strain required to open up the gripper is low at small contact angle where there is minimal interlocking, thus generates small force. At large contact angles, large strains are required for the object to escape the confinement, which corresponds to higher holding force as expected. The simulation results and experimental data are of the same order of magnitude; thus, the computational analysis can serve as a means to evaluate gripping performance.

Pick-and-place experiments were executed to evaluate the gripping performance. Figure 3a illustrates the operation in which objects were gripped, lifted, moved to a target location, and released from the microgripper. Cargo with varying shapes and sizes were microfabricated from a photocurable resin using the same digital maskless lithography technique employed for manufacturing the gripper base. Once the microgel deforms around object, a calcium solution was injected into the experimental container. The shape of the microgel was fixed in seconds, leading to an interlock between the gripper and the cargo (Figure 3b). The cargo was delivered and the shape of the gripper was restored by the addition of $2.5 \mathrm{wt} \%$ EDTA or phosphate buffered solution (PBS) within one to two minutes. PBS contains 0.4 wt $\%$ sodium and potassium ions. The grippers repeatedly transported cargo for more than five times without showing any sign of degradation. The gripping force was increased by raising the temperature of the medium. The contraction of the microgel pinched the surface of the glass microbead 


\section{WILEY-VCH}

horizontally (Figure 3c). We designed a set of experiments to test the relative contributions of fixation of the shape and contraction of the gel on gripping performance. Following the pickup of a glass microbead, the gripper was agitated by moving the holder back and forth several times inside the fluid. The success rate on average of 10 trails was $100 \%$ for cases with both functionalities, while shape-memory only provided $80 \%$ success rate and non-activated gels were unable to keep the cargo. The gripper did not require precise positioning or prefer a certain orientation for the cargo during pick-up (Figure 3d). This adaptability in manipulation also includes cargo with rough surfaces and items significantly heavier than the microgel as demonstrated by transporting metal granules (Figure 3e).

The microgel must reach sufficiently around the sides of the object to be able to provide confinement and secure the pick-up. This requirement may be relaxed if the cargo presents a docking site for engagement (Figure 3f). To test this idea, we fabricated microstructures with a cavity accessible through a slot. The microgel press fit itself into the cavity while the edges of the slot pinched in to the gel (Figure $3 \mathrm{~g}$ ). For this internal gripping operations, thermal compaction of the microgel did not reinforce gripping. On the contrary, by shrinking the size of the microgel, we facilitated undocking and consequently release of cargo. Once mounted on a robotic micromanipulator, the whole operation can be teleoperated using visual feedback (Movie S1). The holder for the gripper base can be integrated with a capillary for the local delivery of calcium and recovery compounds during manipulation (Figure S8). The gripper can sequentially pick up multiple objects from different locations (Figure S9). A common problem encountered during the transport of microscopic objects from one fluid to another is that the disengagement from the fluid surface is quite challenging. At these length scales, surface tension is dominant over inertia and cargo can be easily disengaged from the gripper arms. We could repeatedly operate at the interface and transport objects between media, a feature that is usually required for biomanipulation tasks (Figure S10). 


\section{WILEY-VCH}

The biomanipulation of cell-encapsulating hydrogels has broad applications in regenerative medicine, tissue engineering, and pharmaceutical research. ${ }^{[27]}$ Using soft lithographic templates, we engineered 3D cell-laden microtissues from reconstituted collagen I and 3T3 fibroblasts. The tissues contracted spontaneously and eventually attained a spherical shape with a diameter of $200 \mu \mathrm{m}$ over the course of two days (Figure 4a). The pick-up and transport tasks were executed without causing any plastic deformation to the sample (Figure $4 b$ ). The deformation of microtissues during the manipulation was also minimal and we did not observe a detectable increase in the number of dead cells after the operation. Figure S11 shows the results of the viability assay. Next, we asked whether we could bring multiple microtissues together to create higher-order biological constructs. Once deposited into the same well, cells from different microtissues (labeled with red and green) started to migrate towards each other. This migration and deformation of the surrounding extracellular matrix led to the assembly of microtissues (Figure 4c). The ability to hold and orient biological samples in physiological fluids with high precision can be utilized further to perform localized microsurgical operations. As a proof-ofconcept example, we mounted a glass microcapillary as a second tool and performed dexterous biomanipulation on gripped microtissues for localized injection (Figure 4d). The microgrippers held the microtissues for more than 10 minutes during these operations without showing any sign of fatigue.

The operation of the microgripper does not require a tether provided that the device can be remotely positioned. Untethered, miniaturized robotic devices may enable us to perform minimally invasive operations in complex microenvironments ${ }^{[28]}$ and magnetic fields have been the most widely used strategy for the propulsion of mobile microrobots. ${ }^{[29]}$ We incorporated Nickel microparticles during the fabrication of the base without changing the composition of the microgel end-effector. The magnetic beads were uniaxially aligned using a uniform 


\section{WILEY-VCH}

magnetic field prior to photopolymerization of PEGDA to generate a magnetization profile along the long axis of the gripper (Figure 4e). Magnetic forces are applied directly to the microgripper to control its position and orientation using a robotic manipulation system (Figure S12). We planned a serpentine trajectory, and the microgripper was steered automatically by the system along the path multiple times (Movie S2). Next, we teleoperated a magnetic microgripper to pick-up a polystyrene microbead, transport the cargo along a pre-defined path inside a labyrinth, and finally deliver the cargo to its target destination (Figure 4f). So far, the ambient temperature was raised everywhere in the workspace using a programmable Peltier element for the actuation of the gripper. Previous work has shown that plasmonic photothermal effect of gold nanoparticles efficiently transduces light into localized heat. ${ }^{[30]}$ We synthesized gold nanorods with a longitudinal surface plasmon resonance peak at $785 \mathrm{~nm}$ and incorporated them into the microgel prior to polymerization (Figure 4g). Exposure to near-infrared laser irradiation raised the temperature of the microgel above LCST within 5 seconds, thus actuating the gripper mechanism (Figure $4 \mathrm{~h}$ ). The microgel can be repeatedly activated without a detectable loss of actuation strain (Figure S13). Combined with the magnetic motion control, optical contraction opens up the possibility of operating multiple microgrippers for cooperative missions inside confined space.

We have presented the design, fabrication, and characterization of a universal soft robotic microgripper, and have demonstrated its capability for tethered and untethered micromanipulation of a wide range of different objects including biological samples. The fabrication methodology is compatible with the production of smaller devices (down to tens of micrometers). The surface chemistry of the microgel can be precisely engineered for providing selective adhesion or antifouling. ${ }^{[31]}$ The capability of gently and securely holding microtissues and application of controlled compression has far more implications for biomanipulation than microassembly. Bioactive small molecules can be loaded to the microgel to combine 


\section{WILEY-VCH}

mechanical and chemical conditioning of stem cells or training of immune cells before implantation. Furthermore, the composition and viscoelastic properties of the microgel can be tailored to form a surrogate scaffold or capsule for targeted delivery of cell clusters in vivo.

\section{Experimental Section}

Materials: All reagents were purchased from Sigma Aldrich and used as received, unless otherwise stated. Nickel beads and silver particles were purchased from Good fellow. Gold nanoparticles were synthesized according to a previously described protocol. ${ }^{[30]}$

Fabrication of Microgels: For the synthesis of microgels using shear gels method, a $20 \mu \mathrm{L}$ solution of NIPAM (4.5 wt\%), PEGDA575 crosslinker (0.8 wt\%), Darocure photoinitiator (2 $\mathrm{wt} \%)$ and sodium alginate $(4 \mathrm{wt} \%)$ in deionized water was injected into silicon oil $(1 \mathrm{~mL})$. The suspension was rigorously shaken to form precursor droplets. The droplets were subsequently photopolymerized under UV exposure ( $5 \mathrm{~mW} \mathrm{~cm}^{-2}$, Omnicure 2000) for $2 \mathrm{~min}$. Microgels were purified by washing and resuspending steps in $50 \mathrm{v} / \mathrm{v} \%$ isopropanol aqueous solution and deionized water for 3 times, respectively. Monodisperse suspensions of microgels were prepared using a microfluidic device. Flow focusing microchannels were fabricated using photolithography and replica molding of poly(dimethylsiloxane) PDMS (see Figure S1). The channels were $100 \mu \mathrm{m}$ in height and $200 \mu \mathrm{m}$ in width. Silicon oil was used as the oil phase while the precursor aqueous solution forms the water phase. Size of the microgels were controlled by adjusting the flow rates using a low-pressure microfluidic pump (NEMESYS). The microgels were purified as described above.

Characterization of Microgels: Attenuated total reflection FTIR spectroscopy (Thermo-Fisher Scientific, Nicolet 6700) was used to characterize dehydrated microgels. Force-distance curves of hydrated microgels were obtained using an Atomic Force Microscope (JPK Instruments, Nanowizard III) in force spectroscopy mode by using CP-qp-CONT-PS cantilever (Nanoandmore, Germany) with a spring constant of $0.1 \mathrm{~N} / \mathrm{m}$ and a colloidal tip of $6.1 \mu \mathrm{m}$ in 


\section{WILEY-VCH}

diameter. Microgels were fixed on a petri dish treated with $0.1 \%$ poly-l-lysine solution prior to characterization. Three different microgels were tested for each condition.

Fabrication of microgripper base and cargo units: The base of the gripper was fabricated through a one-step photolithographic process. Microgels were resuspended in a precursor solution that consists of $98 \mathrm{v} / \mathrm{v} \%$ PEGDA575 and $2 \mathrm{v} / \mathrm{v} \%$ Darocure 1173 . The solution was injected into a glass chamber with a thickness determined by microfabricated spacers and placed on the motorized stage of an inverted fluorescence microscope (Nikon, Ti Eclipse). UV light was projected onto the sample with a shape defined by a computer-aided design (CAD)based digital blueprint using a programmable digital mirror display (Andor Technology). The chamber was opened after free-radical polymerization and the microgrippers were immersed in water. The cargo was fabricated using a similar protocol. The precursor solution was replaced by a commercial UV curing adhesive (Norland optical adhesive 81, Thorlabs).

Experimental setup: The temperature inside the chambers was set by a solid-state Peltier module (CP60240, CUI Inc) connected to a programmable PID controller unit (TEC-1089-SV, Meerstetter Engineering). A fiber coupled $785 \mathrm{~nm}$ laser source (Thorlabs) provided NIR illumination. For teleoperated positioning, the microgripper was mounted on a motorized XYZ micromanipulator (SLC-2040, SmarAct GmbH) using a 3D printed adapter. Remotely controlled navigation was performed by using a magnetic manipulation system that consists of two linear translation stages (VT-80, PI) and a rotation stage (DT-34, PI) stacked on top of each other. The stages control the position of a permanent magnet array that pulls the microgripper through magnetic field gradients.

Measurement of gripping force: A spherical-tipped (ruby, $250 \mu \mathrm{m}$ in diameter) commercial MEMS force sensor (FT-S1000, FemtoTools) with a resolution of $0.05 \mu \mathrm{N}$ was mounted on a micromanipulator (Newport NPXYZ100SG) with a custom-machined mount. The force sensor was positioned manually using the corresponding controller (Newport NPC3SG) while being visually monitored using the microscope. The microgripper base was fixed on the surface of a 


\section{WILEY-VCH}

plastic chamber filled with deionized water mounted atop a manual 3-axis stage system (Newport). A long-working distance objective (Mitutoyo 5x) was used to project a magnified image of the microgripper and force sensor onto the imaging sensor of a high-sensitivity camera (Hamamatsu ORCA Flash $4.0 \mathrm{v} 3$ ). The force sensor was powered by a programmable linear power supply (Keysight E3631A), and output was measured using a precision multimeter (Keysight 34465A). Force measurements were obtained using the factory calibration.

Cell culture: All reagents for cell culture were purchased from Thermo Fisher Scientific. NIH 3 T3 fibroblast were cultured in DMEM GlutaMAX supplemented with 10\% fetal bovine serum and $1 \%$ penicillin-streptomycin. Cells were passaged upon achieveing confluency at a 1:4 ratio using $0.25 \%$ Trypsin-EDTA and not kept longer than 20 passages. All experiments were done using cells tested for mycoplasma negative.

Microtissue formation: Microfabricated device with arrays of wells were fabricated from polydimethylsiloxane (PDMS; Sylgard 184, Dow Corning) elastomer using soft lithography. Before cell seeding, the devices were sterilized in $70 \%$ ethanol followed by UV sterilization for $15 \mathrm{~min}$ before treatment with $0.02 \%$ pluronics-F127 (Sigma) solution for $10 \mathrm{~min}$ at room temperature. One million 3T3 fibroblast cells were suspended in $2 \mathrm{mg} \mathrm{ml}^{-1}$ liquid neutralized collagen type I from rat tail (Corning BV Life Sciences) and the suspension was transferred to the device. Cells were spin down into the microwells using centrifugation and excess collagen solution was removed. Cell-laden collagen gels spontaneously compacted and eventually formed spherical microtissues within two days in cell culture medium by incubation at $37^{\circ} \mathrm{C}$.

Fluorescence staining and viability assay: Fibroblasts were stained with the cytoplasmic dyes CellTracker Red CMTPX or CellTracker Green CMFDA (both from Thermo Fisher Scientific) by incubation in $1 \mu \mathrm{M}$ dye dissolved in serum-free DMEM medium for 30 minutes at $37^{\circ} \mathrm{C}$ prior to encapsulation in collagen gel. For life/dead cell staining the Viability/Cytotoxicity Assay Kit (Biotium) was applied according to the manufacturer's protocol. Briefly, a solution 


\section{WILEY-VCH}

of $2 \mu \mathrm{M}$ Calcein AM (green) and $4 \mu \mathrm{M}$ Ethidium homodimer III (red) was prepared in PBS.

Microtissues were incubated inside this solution for 40 minutes at $37^{\circ} \mathrm{C}$.

\section{Supporting Information}

Supporting Information is available from the Wiley Online Library or from the author.

\section{Acknowledgements}

This work was supported by the European Research Council under the ERC grant agreement ROBOCHIP (714609). H. J. gratefully acknowledges Chinese Scholarship Council. We would like to thank the members of the MICROBS Laboratory for their technical support and intellectual feedback. J.M.K. acknowledges Louis David for contributions to the mechanical testing platform.

Received: ((will be filled in by the editorial staff))

Revised: ((will be filled in by the editorial staff)) Published online ((will be filled in by the editorial staff))

References

[1] V. Vitiello, S. L. Lee, T. P. Cundy, G. Z. Yang, IEEE Rev. Biomed. Eng. 2013, 6, 111.

[2] A. W. Feinberg, Annu. Rev. Biomed. Eng. 2015, 17, 243.

[3] C. S. Chen, Trends Cell Biol. 2016, 26, 798.

[4] B. Zhang, A. Korolj, B. F. L. Lai, M. Radisic, Nat. Rev. Mater. 2018, 3, 257.

[5] J. J. Abbott, Z. Nagy, F. Beyeler, B. J. Nelson, IEEE Robot. Autom. Mag. 2007, 14, 92.

[6] D. Rus, M. T. Tolley, Nature 2015, 521, 467.

[7] T. J. Wallin, J. Pikul, R. F. Shepherd, Nat. Rev. Mater. 2018, 3, 84.

[8] L. Ionov, Adv. Funct. Mater. 2013, 23, 4555.

[9] R. M. Erb, J. S. Sander, R. Grisch, A. R. Studart, Nat. Commun. 2013, 4, 1.

[10] D. L. Taylor, M. in het Panhuis, Adv. Mater. 2016, 28, 9060.

[11] X. Hu, J. Zhou, M. Vatankhah-Varnosfaderani, W. F. M. Daniel, Q. Li, A. P. Zhushma, A. V. Dobrynin, S. S. Sheiko, Nat. Commun. 2016, 7, 1.

[12] H. W. Huang, M. S. Sakar, A. J. Petruska, S. Pané, B. J. Nelson, Nat. Commun. 2016, 7, 


\section{WILEY-VCH}

1.

[13] H. Jia, Z. Huang, Z. Fei, P. J. Dyson, Z. Zheng, X. Wang, ACS Appl. Mater. Interfaces 2016, $8,31339$.

[14] E. Diller, M. Sitti, Adv. Funct. Mater. 2014, 24, 4397.

[15] F. Beyeler, A. Neild, S. Oberti, D. J. Bell, Y. Sun, J. Dual, B. J. Nelson, J. Micromechanics Microengineering 2007, 16, 7.

[16] M. Power, A. J. Thompson, S. Anastasova, G. Z. Yang, Small 2018, 14, 1.

[17] E. W. H. Jager, O. Inganäs, I. Lundström, Science. 2000, 288, 2335.

[18] T. Y. Huang, M. S. Sakar, A. Mao, A. J. Petruska, F. Qiu, X. B. Chen, S. Kennedy, D. Mooney, B. J. Nelson, Adv. Mater. 2015, 27, 6644.

[19] H. W. Tung, K. E. Peyer, D. F. Sargent, B. J. Nelson, Appl. Phys. Lett. 2013, 103, 114101.

[20] Z. Ye, M. Sitti, Lab Chip 2014, 14, 2177.

[21] S. Fusco, M. S. Sakar, S. Kennedy, C. Peters, R. Bottani, F. Starsich, A. Mao, G. A. Sotiriou, S. Pané, S. E. Pratsinis, D. Mooney, B. J. Nelson, Adv. Mater. 2014, 26, 952.

[22] T. S. Shim, S. H. Kim, C. J. Heo, H. C. Jeon, S. M. Yang, Angew. Chemie. Int. Ed. 2012, $51,1420$.

[23] J. C. Breger, C. Yoon, R. Xiao, H. R. Kwag, M. O. Wang, J. P. Fisher, T. D. Nguyen, D. H. Gracias, ACS Appl. Mater. Interfaces 2015, 7, 3398.

[24] J. C. Kuo, H. W. Huang, S. W. Tung, Y. J. Yang, Sensors. Actuat. A: Phys. 2014, 211, 121.

[25] D. Rus, M. T. Tolley, Nat. Rev. Mater. 2018, 3, 101.

[26] E. Brown, N. Rodenberg, J. Amend, a. Mozeika, E. Steltz, M. R. Zakin, H. Lipson, H. M. Jaeger, Proc. Natl. Acad. Sci. 2010, 107, 18809.

[27] S. Guven, P. Chen, F. Inci, S. Tasoglu, B. Erkmen, U. Demirci, Trends Biotechnol. 2015, 33, 269.

[28] H. Ceylan, J. Giltinan, K. Kozielski, M. Sitti, Lab Chip 2017, 17, 1705. 


\section{WILEY-VCH}

[29] K. Bente, A. Codutti, F. Bachmann, D. Faivre, Small 2018, 14,1.

[30] Z. Liu, Y. Liu, Y. Chang, H. R. Seyf, A. Henry, A. L. Mattheyses, K. Yehl, Y. Zhang, Z. Huang, K. Salaita, Nat. Methods 2016, 13, 143.

[31] M. J. Webber, E. A. Appel, E. W. Meijer, R. Langer, Nat. Mater. 2015, 15, 13. 


\section{WILEY-VCH}
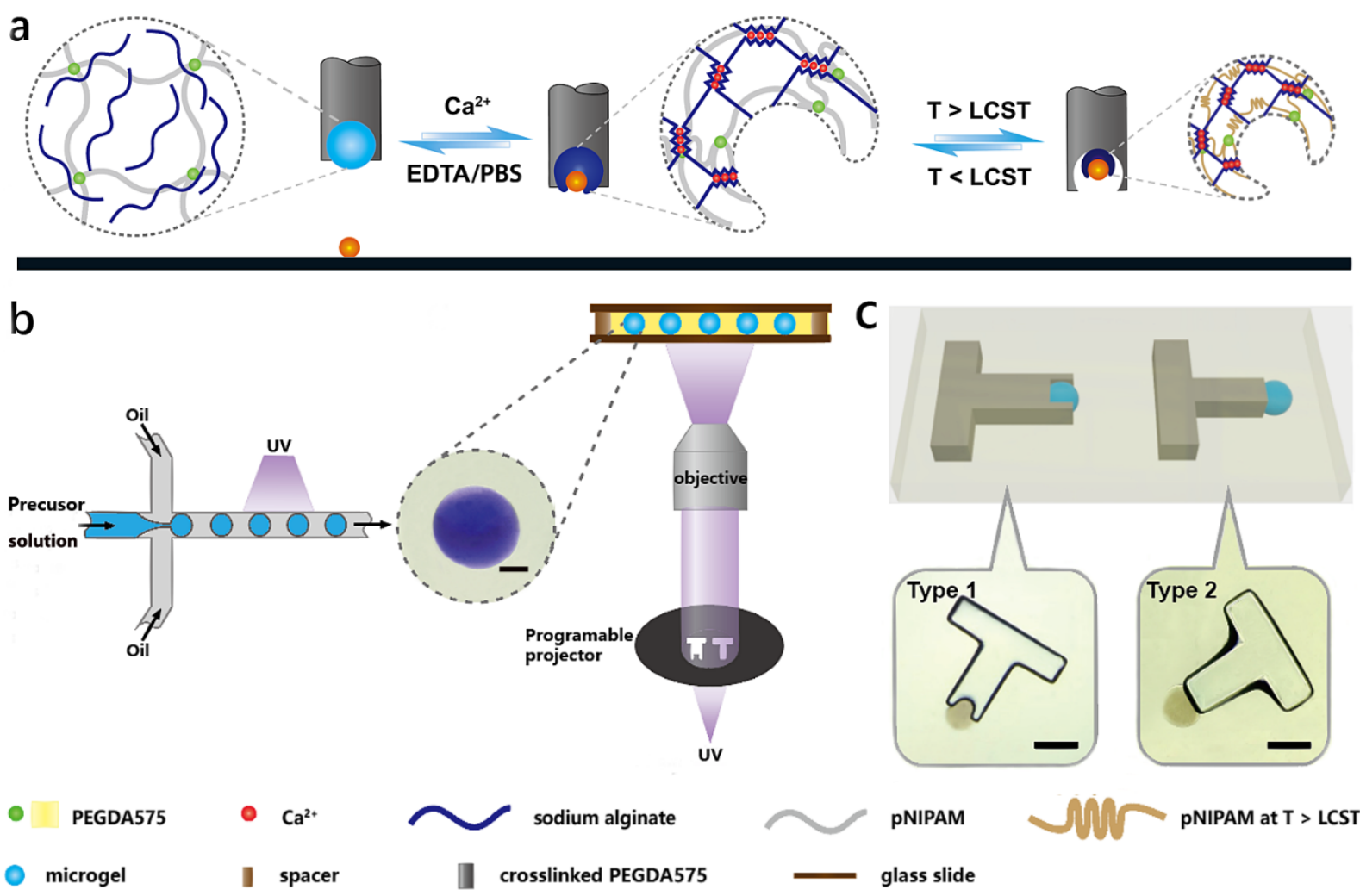

Figure 1. Concept and realization of universal soft robotic microgripper systems. a) Schematic illustration of the gripping mechanism based on reversible shape-memory and thermoresponsive swelling properties of the synthesized hydrogel. b) Fabrication of microgrippers using microfluidics and maskless photolithography. The microgels were coupled to the PEGDA base via in situ polymerization. Scale bar, $200 \mu \mathrm{m}$. c) Two types of microgrippers were designed for the grasping of objects from their exterior (Type I) or through an internal cavity (Type II). Lower panel shows top view images of the microgrippers. Scale bars, $500 \mu \mathrm{m}$. 


\section{WILEY-VCH}
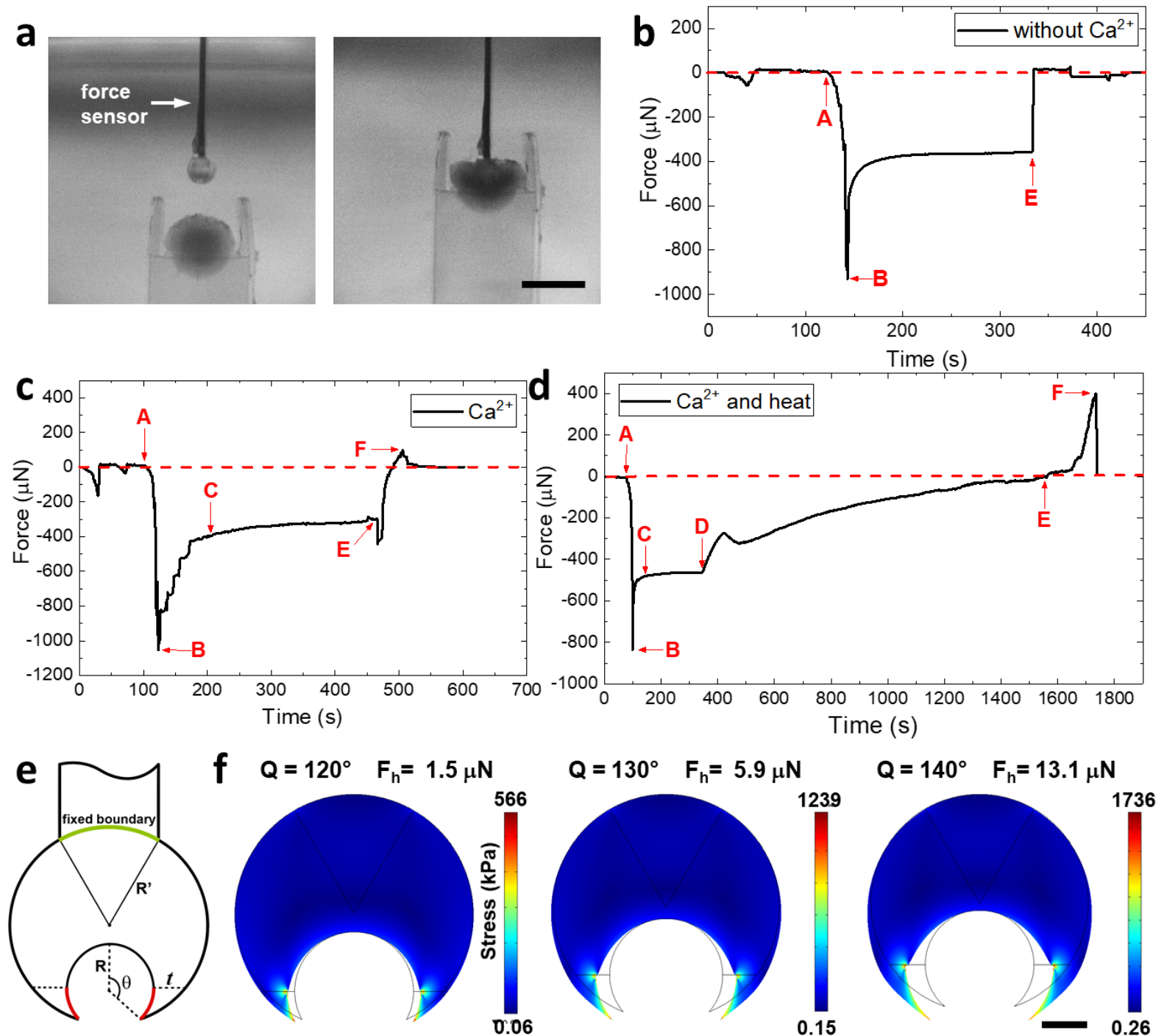

Figure 2. Gripper holding force. a) Optical images of the MEMS force sensor approaching the gripper and compressed gripper fully encapsulating the sensor tip. Scale bar, $500 \mu \mathrm{m}$. MEMS force readings from experiments during which a compression-decompression protocol was applied b) without initiating shape-memory or thermal contraction, c) the shape was fixed with the addition of $5 \mathrm{wt} \% \mathrm{Ca}^{2+}$ ions, and $\mathrm{d}$ ) the shape was fixed and the temperature was raised to actuate the microgel. Capital letters along with arrows indicate the time points for the beginning of compression of the gel (A), end of compression (B), addition of $5 \mathrm{wt} \% \mathrm{Ca}^{2+}$ ions (C), raising the temperature above LCST (D), beginning of the decompression of the gel (E) and detachment of the sensor tip from the gel (F), respectively. e) Sketch of the microgripper denoting the essential geometric parameters and boundary conditions. The strain was applied normal to the gripper arm (shown in red) in simulations. f) Finite element simulations of the bending stress for different contact angles. The arms were displaced by the amount required to release the cargo. Scale bar, $100 \mu \mathrm{m}$. 


\section{WILEY-VCH}

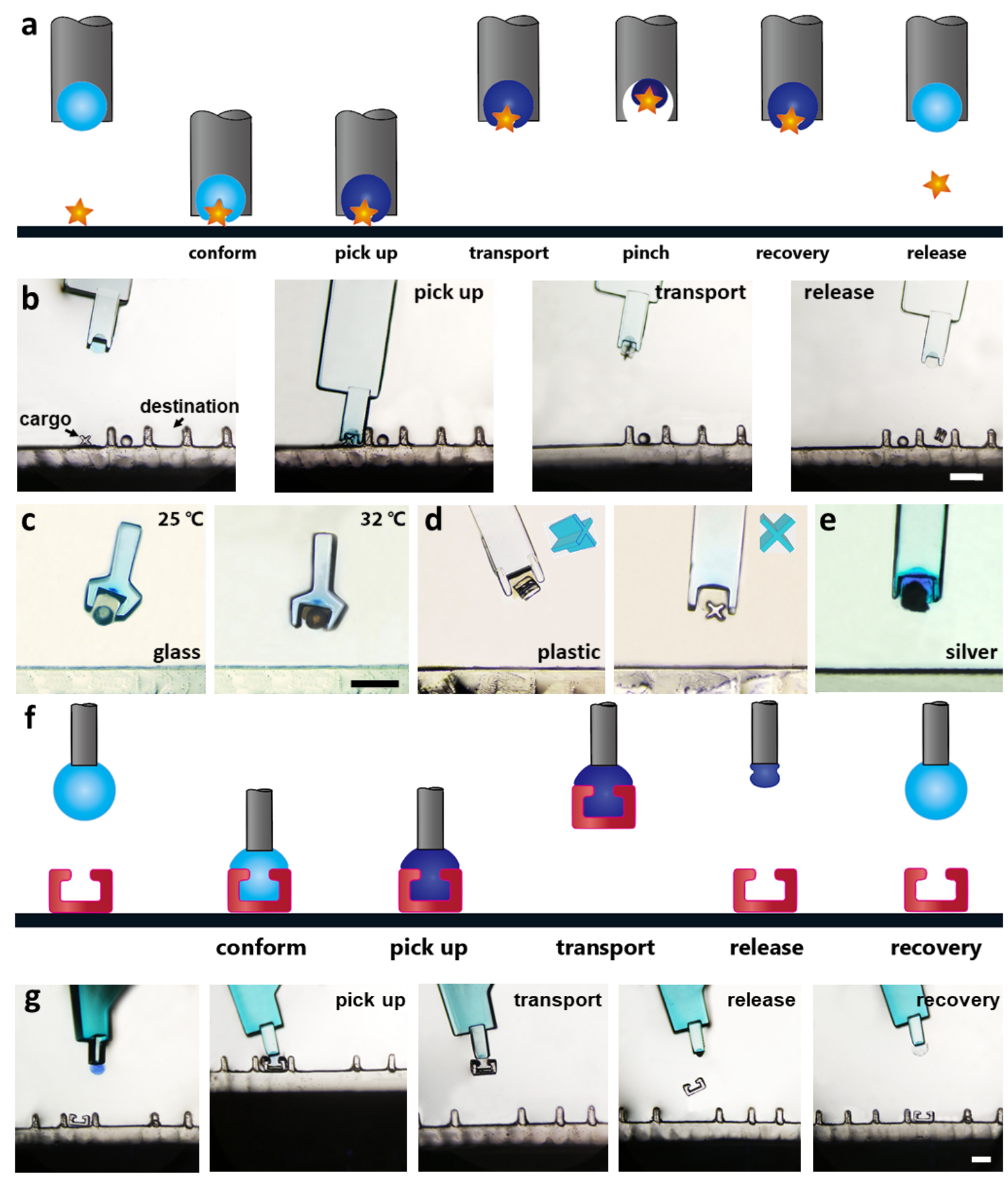

Figure 3. Demonstration of transport of a wide range of microscopic objects without the need for active feedback. a) Schematic description for gripping objects by encapsulating them inside the microgel. b) Optical images from a representative experiment. Scale bar, $500 \mu \mathrm{m}$. c) Pinching microgel tightens the grip and, thus increases the holding force. d) The orientation of the cargo is not important for successful pick-up as long as the gel can mold itself around. e) Gripper can hold objects significantly heavier than its own weight. f) Schematic description for gripping objects by holding them from an extremity. g) Optical images from a representative experiment. Scale bar, $500 \mu \mathrm{m}$. 


\section{WILEY-VCH}

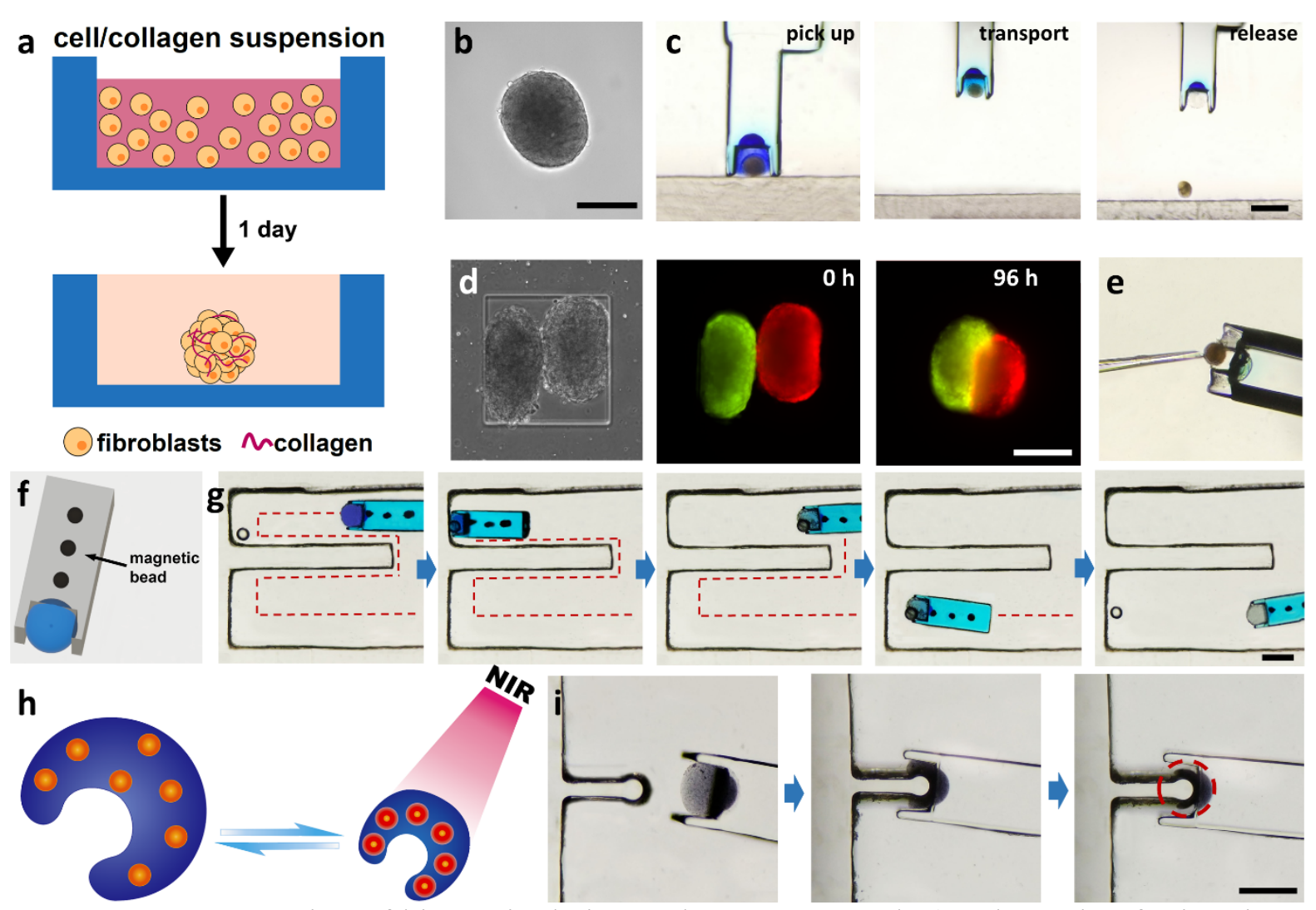

Figure 4. Demonstration of biomanipulation and remote control. a) Schematic of microtissue device and workflow. b) Micrograph of microtissue before transport. Scale bar, $200 \mu \mathrm{m}$. c) Optical images from a representative microtissue transport trial. d) Microtissues are transported into the same well. Staining for cytoplasm with different colors showing the merge of tissues in time. Scale bar, $200 \mu \mathrm{m}$. e) Dexterous manipulation of microtissue held by the microgripper using teleoperated surgical tools. f) Schemetic showing gripper with magnetic base. g) Magnetic microgripper being remotely navigated inside a maze to pick-up, transport, and deliver a glass microbead. Scale bar, $200 \mu \mathrm{m}$. h) Schematic illustration of microgel contraction triggered by NIR illumination. Gold nanorods (orange) transduce light into heat and raise the temperature above LCST. i) The microgel contracts around the fixed target object upon NIR illumination. The area of laser exposure is marked with red circle. Scale bar, $500 \mu \mathrm{m}$. 